\title{
Vibration-assisted resonance in photosynthetic excitation-energy transfer
}

\author{
E. K. Irish, ${ }^{1, *}$ R. Gómez-Bombarelli, ${ }^{2}$ and B. W. Lovett ${ }^{1,3, \dagger}$ \\ ${ }^{1}$ SUPA, Department of Physics and Astronomy, University of St Andrews, St Andrews KY16 9SS, United Kingdom \\ ${ }^{2}$ Department of Chemistry and Chemical Biology, Harvard University, Cambridge, Massachusetts 02138, USA \\ ${ }^{3}$ Department of Materials, University of Oxford, Oxford OX1 3PH, United Kingdom
}

(Received 3 February 2014; published 14 July 2014)

\begin{abstract}
Understanding how the effectiveness of natural photosynthetic energy-harvesting systems arises from the interplay between quantum coherence and environmental noise represents a significant challenge for quantum theory. Recently it has begun to be appreciated that discrete molecular vibrational modes may play an important role in the dynamics of such systems. Here we present a microscopic mechanism by which intramolecular vibrations may be able to contribute to the efficiency and directionality of energy transfer. Excited vibrational states create resonant pathways through the system, supporting fast and efficient energy transport. Vibrational damping together with the natural downhill arrangement of molecular energy levels gives intrinsic directionality to the energy flow. Analytical and numerical results demonstrate a significant enhancement of the efficiency and directionality of energy transport that can be directly related to the existence of resonances between vibrational and excitonic levels.
\end{abstract}

DOI: 10.1103/PhysRevA.90.012510

PACS number(s): 31.70.Hq, 03.65.Yz, 87.15.hg, 82.20.-w

\section{INTRODUCTION}

Photosynthetic organisms have evolved a system of lightharvesting antenna complexes that absorb energy from sunlight and funnel it into a reaction center where the captured light energy is converted into stored chemical energy [1]. For this extended system to function effectively, energy transfer through the antenna to the reaction center must be efficient, in the sense that energy is transferred from molecule to molecule with high probability. The flow of energy also needs to be preferentially directed toward the reaction center. Understanding how such efficient and directional energy transport arises in natural photosynthetic systems is currently a major area of research.

Two theoretical approaches to studying energy transport are usually distinguished. When the coupling between molecules in a network is weak compared to their interaction with the environment, energy transfer is incoherent and can be described as an effectively classical "hopping" process with rates determined by the Fermi golden rule. The opposite limit is the coherent or exciton approach, wherein the molecules are strongly coupled and interact only weakly with their environment. A major theoretical challenge is presented by the fact that many photosynthetic systems, such as the much-studied Fenna-Matthews-Olson complex, fall into an intermediate regime.

In the incoherent Förster resonance energy transfer (FRET) model, the conditions for efficient and directional energy transfer are well understood [2]. The FRET mechanism can facilitate efficient energy transfer only between resonant energy levels. In photosynthetic pigment-protein complexes (PPCs), however, chromophores are typically arranged so that their energy levels form a downhill gradient or "energy funnel" [1]. Such disordered systems can still support efficient energy transfer thanks to the existence of nuclear vibrational

\footnotetext{
*eki2@st-andrews.ac.uk

†bw14@st-andrews.ac.uk
}

sidebands. Resonant transfer occurs between a donor molecule and a vibrationally excited state of a lower-energy acceptor molecule. The vibrational excitations decay on a relatively fast timescale, creating a difference between the excitation and fluorescence frequencies of the molecule known as the Stokes shift. The Stokes shift favors downhill energy transfer, producing directionality in the FRET mechanism.

Although nuclear vibrational modes are central to producing efficient one-way energy transport in the incoherent FRET theory, coherent models of energy transfer are usually concerned with the electronic degrees of freedom alone. Vibrations, whether they originate from intramolecular nuclear motion or motions of the protein and solvent environment, are reduced to a collective environment or "bath" which can then be treated at various levels of approximation. In the simplest case, often termed dephasing-assisted transport (DAT) [3] or environment-assisted quantum transport (ENAQT) [4], the primary effect of the bath is to dephase electronic coherences between different molecules. Studies of this model have produced the important and seemingly counterintuitive insight that environmental noise can improve energy transport in disordered quantum networks [3-8].

As in the incoherent case, efficient energy transfer in coherent models requires resonance between molecules. Consider the simple case of two sites. Fully coherent Hamiltonian dynamics can only produce full population transfer from one site to the other if the two sites have identical energies. Once the energy difference exceeds the coupling, the amplitude of population oscillations is significantly reduced and the excitation becomes primarily localized on a single site. The localization effects of quantum coherent dynamics in disordered systems can be overcome to some extent by the addition of dephasing. The environmentally induced energy fluctuations responsible for dephasing can momentarily bring the energies of two adjacent sites into resonance, allowing efficient transfer between them. However, since the energy gaps between adjacent chromophores can vary substantially, the individual dephasing rates required to optimize transport without completely destroying coherence must also vary 
substantially among chromophores. A study on the FennaMatthews-Olson complex (FMO) using the DAT model in which the transfer probability was maximized with respect to individual site dephasing rates found that the optimal dephasing rate for each chromophore varied over two orders of magnitude depending on its location in the complex [3]; subsequent work gave similar results [6]. In a highly structured PPC such as FMO, where the average chromophore separation is on the order of $1.2 \mathrm{~nm}$ [9], it seems unlikely that adjacent chromophores experience such drastically different noise levels. Recent quantum chemistry calculations indicate that the strength of the interaction with the environment does depend on the position of the chromophore in the FMO complex, but the values differ only by a factor of two to three [10].

Producing one-way energy transport in coherent models is more difficult. Fully coherent Hamiltonian evolution is inherently reversible. Open-quantum-system techniques overcome this problem by taking the quantum system under study to be coupled to a large environment, leading to effectively irreversible evolution. Such evolution may or may not appear as a directional, coherent energy-transfer process. Pure dephasing in the site basis, as in DAT-ENAQT models, results in a diffusive process whose limiting distribution is equal population on each site in the system [4]. In order to achieve one-way energy transport, these models rely on the addition of a trap site to which energy is transferred irreversibly at a constant rate. This mechanism is designed to model exciton transfer from peripheral complexes to the reaction center [5]. In the case of the much-studied FMO complex, there is in fact very little experimental data on which to base the trapping model [see 41, and references therein]. Furthermore, the key experiments on energy-transfer dynamics in FMO were carried out on purified complexes that do not contain reaction centers, and thus any observed directionality must originate from a different mechanism.

The Stokes shift together with the downhill arrangement of energy levels provides directionality in the incoherent FRET model. However, the level of approximation employed in deriving pure dephasing models eliminates the Stokes shift. The Lindblad master equation with pure dephasing is equivalent to the stochastic Haken-Strobl model [4]. The latter corresponds to the fast modulation or high-temperature limit, in which the bath a has zero correlation time [11], and the Stokes shift vanishes in this limit [12]. Leegwater [5] has further shown that coherent dynamics with pure dephasing is identical to the incoherent hopping predicted by FRET when the interaction with the environment becomes much larger than the coherent coupling between individual molecules. In the high-temperature limit required for the bath correlation time to vanish, the FRET rates for forward and backward energy transfer become equal and there is no longer a preferred transfer direction.

Recent work, both experimental and theoretical, has shown that vibrations in PPCs do not behave like a simple thermal bath with a smooth spectral density. Vibrational spectroscopy has revealed rich vibrational structures in FMO [13,14] and other photosynthetic complexes [15,16]. Molecular dynamics simulations of FMO have likewise shown that the spectral density contains a number of distinct peaks that can be attributed to intramolecular nuclear vibrational modes $[17,18]$. Some recent numerical studies have suggested that these modes may play a substantial role in coherent energy transfer [7,19-23], particularly as a number of vibrational frequencies lie within the range of exciton energy splittings and resonance effects may therefore be important [8,20-22,24-26]. However, the microscopic mechanisms by which vibrations can contribute to energy transfer are not well understood and the specific role of resonance has yet to be conclusively demonstrated.

The theory introduced here provides an alternative approach to satisfying the conditions of resonance and directionality, by including nuclear vibrational modes explicitly in the coherent part of the system under study. As in FRET, resonant transfer occurs between a donor molecule and a vibrationally excited state of the acceptor molecule. Subsequent decay of the vibrational excitation effectively produces a Stokes shift, creating a preferential direction of transfer. However, in our model both the electronic states and the relevant vibrational states are treated coherently within an exciton approach. The environmental interaction is still described by a Lindblad master equation, as in DAT-ENAQT-type models, retaining much of the simplicity of pure dephasing models while incorporating critical features of nuclear vibrational coupling.

Our motivation is not to compete with formally exact numerical techniques such as those in Refs. [10,17,1921,23,27-29]. Rather, we are interested in understanding, in an intuitive way and at a microscopic level, how the sharp peaks in the structure of the environmental spectral density may play a role in energy-transfer dynamics. That insight, in turn, may help direct work using the more accurate but much more computationally demanding numerical techniques.

\section{COHERENT MODEL OF VIBRATION-ASSISTED RESONANCE}

The starting point for our theory is the standard electronic Hamiltonian $H_{\mathrm{el}}$ used in exciton theory [30]. In the site basis, the electronic Hamiltonian is given by

$$
H_{\mathrm{el}}=\sum_{i=1}^{N} \epsilon_{i}|i\rangle\left\langle i\left|+\sum_{i \neq j} J_{i j}\right| i\right\rangle\langle j|,
$$

where $N$ is the number of chromophores or sites, $|i\rangle$ denotes the electronic excited state of molecule $i, \epsilon_{i}$ is the electronic excitation energy of $|i\rangle$ relative to its ground state $\left|g_{i}\right\rangle$, and $J_{i j}$ is the excitonic coupling between sites $i$ and $j$.

To the electronic Hamiltonian we add two additional terms $H_{\text {vib }}$ and $H_{\text {el-vib }}$ to describe the vibrational modes and their coupling to the electronic states, respectively. The relevant vibrational modes are intramolecular vibrations excited by the electronic transition from the ground state to an excited state. Intermolecular vibrations, in which all molecules couple to a common vibrational mode, are known to assist energy transfer (see Refs. [24,31,32], to name but a few); furthermore, correlations of energy fluctuations in the site basis have been posited as an explanation for the discrepancy between calculated and observed dephasing times (see Refs. [14,22] of Ref. [29]). However, detailed atomistic simulations of the FMO complex have found no evidence for such correlations $[17,18,29]$. Furthermore, intramolecular vibrations have a well-understood physical and chemical basis and the inclusion 
of these vibrations provides a direct link between the exciton formalism and the FRET mechanism.

Intramolecular vibrational modes arise from the motions of nuclei within a nonlinear polyatomic molecule [33]. These molecular vibrations are usually described within the harmonic approximation as small displacements on a parabolic potential-energy surface around an equilibrium position. When a chromophore absorbs an incoming photon, an electron is promoted from an occupied low-energy molecular orbital to an unoccupied one with higher energy. The excitation is much faster than the nuclear response and thus, within the Franck-Condon approximation, it is assumed that nuclei remain static during the optical excitation and afterwards relax to the excited-state equilibrium geometry. To lowest order, the excited-state normal modes can be approximated by the same parabolic potential-energy surfaces as in the ground state but with a displaced equilibrium position. A similar process occurs during fluorescence emission: the nuclei remain static during the transition-in this case at the equilibrium geometry of the excited electronic state-and after the change in electronic state, vibrational modes mediate the relaxation to the ground-state equilibrium geometry. Experimentally, the energy difference between absorption and emission peaks in the spectra is termed the Stokes shift.

The vibrational Hamiltonian consists of $n(i)$ vibrational modes of each of the $i$ molecules:

$$
H_{\mathrm{vib}}=\sum_{i=1}^{N} \sum_{k=1}^{n(i)} \omega_{i k} a_{i k}^{\dagger} a_{i k},
$$

where $a_{i k}^{\dagger}\left(a_{i k}\right)$ is the raising (lowering) operator for the mode with frequency $\omega_{i k}$. The zero-point energy has been omitted and $\hbar$ has been set to 1 . Each vibrational mode is linearly coupled with strength $\lambda_{i k}$ to the excited state of the chromophore on which the vibration is localized, giving

$$
H_{\mathrm{el}-\mathrm{vib}}=\sum_{i=1}^{N} \sum_{k=1}^{n(i)} \lambda_{i k}\left(a_{i k}^{\dagger}+a_{i k}\right)|i\rangle\langle i| .
$$

We have assumed that the Franck-Condon approximation is valid and that the ground- and excited-state modes differ only by a displacement in equilibrium position. Within this approximation the transition dipole moment is assumed to be independent from the nuclear coordinates and the vibrational frequencies are assumed to remain unchanged after the excitation. For bacteriochlorophyll $a$, results at the time-dependent density functional theory (TD-DFT) level of theory suggest that, for those modes coupled to the electronic excitation, the differences between the excited-state frequency and the average frequency of ground-state motions are all very small [34]. Written in the ground-state basis, the displacement of the corresponding excited-state vibrational mode appears as a linear coupling term.

Interactions with the environment, which is comprised of protein vibrational modes and solvent fluctuations, are incorporated by means of a Markovian master equation [35]:

$$
\frac{d \rho}{d t}=-i[H, \rho(t)]-\mathcal{L}_{\mathrm{deph}}(\rho(t))-\mathcal{L}_{\mathrm{vib}}(\rho(t)),
$$

where $H=H_{\mathrm{el}}+H_{\mathrm{vib}}+H_{\mathrm{el}-\mathrm{vib}}$ and $\rho(t)$ is the density matrix of the full system of electronic states and nuclear vibrational modes. Dephasing of electronic coherences in the site basis is described by the Lindblad superoperator

$$
\mathcal{L}_{\text {deph }}(\rho(t))=\sum_{i=1}^{N} \gamma_{i}^{\text {deph }}\left(\frac{1}{2}\left\{L_{i}^{\dagger} L_{i}, \rho\right\}-L_{i} \rho L_{i}^{\dagger}\right),
$$

where $L_{i}=\left|g_{i}\right\rangle\left\langle g_{i}|-| i\right\rangle\langle i|$. Here $\{A, B\}=A B+B A$ denotes the anticommutator, $\left|g_{i}\right\rangle$ is the ground state of site $i$, and $\gamma_{i}^{\text {deph }}$ is the electronic dephasing rate of site $i$. Damping of the nuclear vibrational modes is also needed in order to incorporate a Stokes-shift-like effect into the exciton model and is given by

$$
\begin{aligned}
\mathcal{L}_{\mathrm{vib}}(\rho(t))= & \sum_{i=1}^{N} \sum_{k=1}^{n(i)} \gamma_{i k}^{\mathrm{vib}}\left[\left(v_{i k}+1\right)\left(\left\{a^{\dagger} a, \rho\right\}-2 a \rho a^{\dagger}\right)\right. \\
& \left.+v_{i k}\left(\left\{a a^{\dagger}, \rho\right\}-2 a^{\dagger} \rho a\right)\right] .
\end{aligned}
$$

Here, $\gamma_{i k}^{\mathrm{vib}}$ is the damping rate of mode $i k$ and $v_{i k}$ is the number of thermal excitations in mode $i k$ in the steady state. We have omitted decay of the electronic excitations since we are interested in dynamics on time scales much shorter than the electronic decay time, but this effect is easily incorporated within the master-equation formalism.

While the model we have constructed retains the conceptual and computational simplicity of the standard Lindblad master-equation formalism, it goes beyond the limitations of the usual exciton models in two ways. The first is in its treatment of temperature dependence. As discussed in the introduction, the restriction of the electronic dissipation to pure dephasing processes is equivalent to taking the hightemperature limit. However, in our study we additionally take into account the temperature dependence of the vibrational dissipation processes. While this sounds inconsistent at first, it is perhaps not entirely unjustifiable. In the vibrational spectroscopy experiments of Wendling et al., the maximum of the background phonon distribution was assigned to be around $20 \mathrm{~cm}^{-1}$. The exciton splittings in FMO, which correspond to the vibrational frequencies of interest in our model, are on the order of 100 to $500 \mathrm{~cm}^{-1}$. This order-of-magnitude separation in frequency between the phonons and the intramolecular vibrational modes suggests that finite-temperature effects are more important for the vibrational modes. Second, by treating discrete intramolecular vibrational modes within the system Hamiltonian rather than within the spectral density of the bath, the model incorporates a certain degree of nonMarkovian behavior. Excitations can be exchanged between the electronic system and the environmental fluctuations represented by the intramolecular vibrations, within a timescale determined by the electronic dephasing and vibrational decay rates.

In realistic biological systems, the addition of intramolecular vibrational modes to the system Hamiltonian rapidly expands the system size beyond the limits of computational feasibility. Even a system as small as FMO has around thirty strong vibrational modes $[13,14]$, making a full system treatment intractable. However, we argue here that only the vibrational levels that create resonant pathways through the 


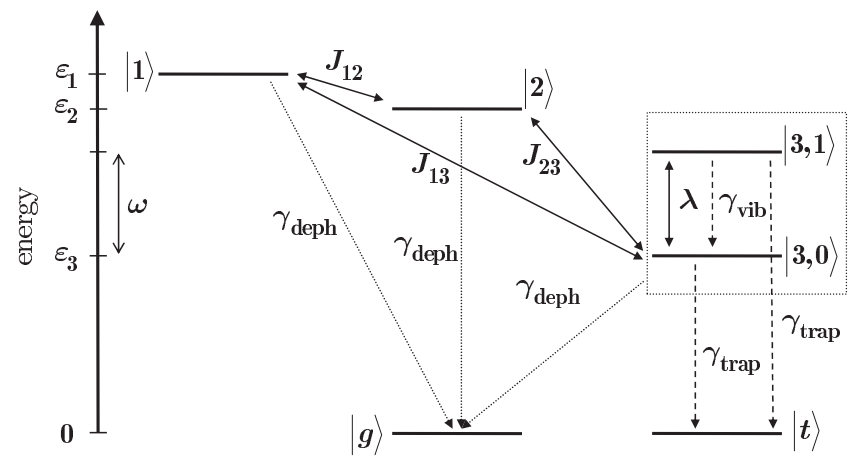

FIG. 1. Diagram of the three-site model, with site 3 coupled to a vibrational mode that is restricted to either 0 or 1 excitation(s). Solid arrows indicate coherent couplings, dashed arrows indicate irreversible decay pathways, and dotted arrows indicate dephasing pathways.

network contribute substantially to the coherent dynamics of energy transfer. With this restriction the system size can be substantially reduced while retaining the essential physics.

\section{ANALYSIS OF A SIMPLE CASE}

To illustrate the vibration-assisted resonance mechanism we construct a minimal model, based on sites 1-3 of the FMO complex, in which the relevant physical effects can be clearly seen. To a good approximation, these three sites comprise one of two energy-transfer branches identified in FMO [36,37]. The model, shown in Fig. 1, consists of three sites, whose electronically excited states are denoted by $|i\rangle, i=\{1,2,3\}$; the overall ground state of the system is denoted by $|g\rangle$. Sites 1 and 2 are near resonance and strongly coupled, while site 3 has a much smaller excitation energy and is more weakly coupled to the other sites. For simplicity we include only a single vibrational mode of frequency $\omega$, linearly coupled with strength $\lambda$ to site 3 . Furthermore, we restrict the mode to its ground and first-excited states; these states are denoted, respectively, by $|3,0\rangle$ and $|3,1\rangle$. Equations (1)-(3) then reduce to

$$
\begin{aligned}
H= & \epsilon_{1}|1\rangle\left\langle 1\left|+\epsilon_{2}\right| 2\right\rangle\left\langle 2\left|+\epsilon_{3}\right| 3,0\right\rangle\langle 3,0| \\
& +\left(\epsilon_{3}+\omega\right)|3,1\rangle\langle 3,1|+J_{12}(|1\rangle\langle 2| \\
& +|2\rangle\langle 1|)+J_{23}(|2\rangle\langle 3,0|+| 3,0\rangle\langle 2|)+J_{13}(|1\rangle\langle 3,0| \\
& +|3,0\rangle\langle 1|)+\lambda(|3,0\rangle\langle 3,1|+| 3,1\rangle\langle 3,0|) .
\end{aligned}
$$

The dephasing rate $\gamma_{\text {deph }}$ is assumed to be the same for each site, and the vibrational mode decays from its excited state $|3,1\rangle$ to its ground state $|3,0\rangle$ with rate $\gamma_{\text {vib. In order to compare }}$ our results directly with those from DAT-ENAQT models we add a trapping state $|t\rangle$ that absorbs energy from both vibronic levels on site 3 at rate $\gamma_{\text {trap }}$. We also consider the case without the trap and show that it is not, in fact, necessary to produce directional energy transfer. For simplicity we neglect losses from exciton recombination or other nonradiative decay processes which occur on nanosecond timescales [9], much slower than the 0.5 to 5 ps timescales we are interested in.
TABLE I. Parameter values in $\mathrm{cm}^{-1}$ for Prosthecochloris aestuarii, taken from Ref. [36].

\begin{tabular}{lrrr}
\hline \hline \multicolumn{2}{c}{ Site energies } & \multicolumn{2}{c}{ Couplings } \\
\hline$\epsilon_{1}$ & 12475 & $J_{12}$ & -98.2 \\
$\epsilon_{2}$ & 12460 & $J_{13}$ & 5.4 \\
$\epsilon_{3}$ & 12225 & $J_{23}$ & 30.5 \\
\hline \hline
\end{tabular}

To ensure that our model is both physically motivated and realistic, parameter values used in the discussion of the model and numerical simulations are drawn from experimental results wherever possible. Exact values for the site energies and excitonic couplings vary depending on the organism being studied and the details of experimental spectra and theoretical fitting methods [9]. However, certain trends are clear. The relations $\epsilon_{1} \sim \epsilon_{2}>\epsilon_{3}, J_{12}>J_{23}>J_{13}, J_{12} \gg$ $\left|\epsilon_{1}-\epsilon_{2}\right|$, and $\epsilon_{2}-\epsilon_{3} \gg J_{23}$ are useful in obtaining physical insight into the model. For numerical calculations we use site energies and excitonic couplings from Ref. [36], as shown in Table I.

The magnitude of the vibrational coupling constant $\lambda$ requires careful consideration. This term originates from the shift of the nuclear normal-mode potential-energy surface upon electronic excitation of a pigment molecule [30,38], from which $\lambda=\omega \sqrt{S_{\omega}}$, where $S_{\omega}$ is the Huang-Rhys factor of the vibrational mode with frequency $\omega$. Adolphs and Renger [36] considered a vibrational spectral density consisting of a broad continuous background with a single high-frequency mode at $\omega_{H}=180 \mathrm{~cm}^{-1}$. Comparing this model with the experimental vibrational spectra of Wendling et al. [13], they estimated the Huang-Rhys factor $S_{H}=0.22$. Subsequent work has used the same or similar values [7,8]. However, the Huang-Rhys factors for individual modes found in the experiments of Wendling et al. are on the order of $S_{\omega} \lesssim 0.01$, and in fact the sum of the Huang-Rhys factors for the thirty measured vibrational modes is on the order of $S_{\text {tot }} \sim 0.25$ [13]. As the physics presented here is based on a resonance mechanism and therefore relies on close frequency matching, we choose the Huang-Rhys factor $S_{\omega} \sim 0.01$ appropriate to an individual mode rather than the value $S \sim 0.2$ that corresponds to an effective coupling to a large number of modes. For the frequencies of interest in our model this yields $\lambda \sim 15 \mathrm{~cm}^{-1}$, so that $\lambda<J_{23}<J_{12}$.

The first step in our analysis is to identify the resonances in the system and their effect on the system in the absence of any environmental interactions. From the relations among the various energies and coupling strengths discussed above, we can extract a simplified picture that provides good intuition for the physics of the model. States $|1\rangle$ and $|2\rangle$ are near resonance and strongly coupled, so they must be treated in the delocalized "exciton basis." This produces the set of basis states and couplings illustrated in Fig. 2: the new states $| \pm\rangle$ with energies $\epsilon_{ \pm}$are the excitonic states spanning the $\{|1\rangle,|2\rangle\}$ subspace, $J_{ \pm 3}$ denotes their couplings to state $|3,0\rangle$, and the coupling $\lambda$ between $|3,0\rangle$ and $|3,1\rangle$ remains unchanged. For our system $\left|J_{ \pm 3}\right| \gtrsim \lambda$ and all three couplings are small enough to be treated as perturbative parameters. As long as $\omega$ is chosen so that $|3,1\rangle$ does not come into resonance with $| \pm\rangle$, the eigenstates will not vary significantly from the basis states. 


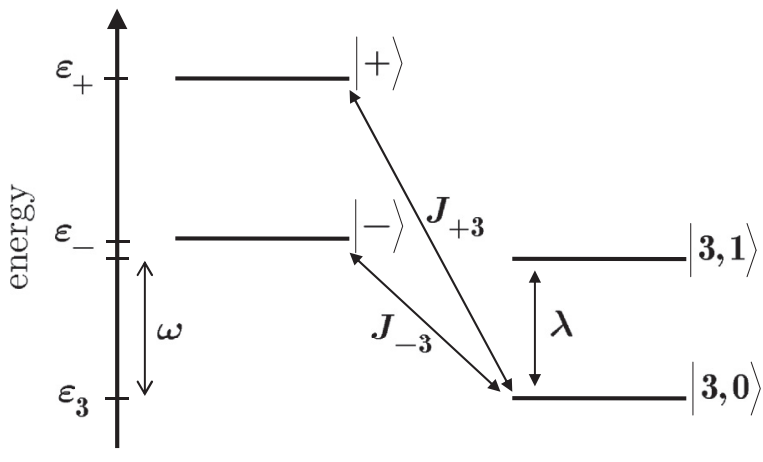

FIG. 2. Simplified model illustrating the resonance mechanism within a perturbative framework. The delocalized excitonic states $| \pm\rangle$ diagonalize the $\{|1\rangle,|2\rangle\}$ subspace. The transformed couplings $J_{ \pm 3}$ are of similar magnitude to $\lambda$, which is much smaller than $\epsilon_{ \pm}-\epsilon_{3}$. When $\omega \simeq \epsilon_{ \pm}-\epsilon_{3}$, a resonance occurs and the exciton state $| \pm\rangle$ becomes strongly mixed with the vibrationally excited state $|3,1\rangle$ even though the states are only coupled to second order (via the intermediate state $|3,0\rangle)$ and both $J_{ \pm 3}$ and $\lambda$ are small.

However, when $\omega \simeq \epsilon_{ \pm}-\epsilon_{3}$ a resonance is created and $|3,1\rangle$ can become strongly mixed with $| \pm\rangle$.

The effect of the resonance may be analyzed within this picture using degenerate perturbation theory. The Hamiltonian is divided into a "bare" term $H_{0}$ and a perturbation term $H^{\prime}$ :

$$
\begin{gathered}
H=H_{0}+H^{\prime}, \\
H_{0}=\epsilon_{+}|+\rangle\left\langle+\left|+\epsilon_{-}\right|-\right\rangle\left\langle-\left|+\epsilon_{3}\right| 3,0\right\rangle\langle 3,0| \\
+\left(\epsilon_{3}+\omega\right)|3,1\rangle\langle 3,1|, \\
H^{\prime}=J_{+3}(|+\rangle\langle 3,0|+| 3,0\rangle\langle+|) \\
+J_{-3}(|-\rangle\langle 3,0|+| 3,0\rangle\langle-|) \\
+\lambda(|3,0\rangle\langle 3,1|+| 3,1\rangle\langle 3,0|) .
\end{gathered}
$$

For notational simplicity we will take the resonant pair of states to be $|-\rangle$ and $|3,1\rangle$, so that $\omega=\epsilon_{-}-\epsilon_{3}$. The resulting degeneracy in $H_{0}$ creates divergences in the series expansions of the eigenstates of $H$. Therefore, the first step in applying perturbation theory must be to identify the appropriate superpositions of the resonant states that will remove the degeneracy.

Looking at Eq. (8) it is immediately evident that there is no matrix element of $H^{\prime}$ that directly connects the two resonant states, so the degeneracy cannot be removed by simply diagonalizing the degenerate subspace of $H$. It is necessary, then, to work to second order in the expansion of the eigenstates and energies [39] to determine an effective perturbation Hamiltonian $\tilde{H}$ in the $\{|-\rangle,|3,1\rangle\}$ subspace. For two states $|m\rangle$ and $|l\rangle$ with $E_{m}=E_{l}$, the general form of $\tilde{H}$ may be expressed as

$$
\tilde{H}=\tilde{H}_{m m}|m\rangle\left\langle m\left|+\tilde{H}_{l l}\right| l\right\rangle\langle l|+\left(\tilde{H}_{m l}|m\rangle\langle l|+\text { H.c. }\right),
$$

where H.c. denotes the Hermitian conjugate and the matrix elements are given by

$$
\begin{gathered}
\tilde{H}_{m m}=\sum_{n \neq l, m} \frac{\left|\left\langle n\left|H^{\prime}\right| m\right\rangle\right|^{2}}{E_{m}-E_{n}}, \\
\tilde{H}_{l l}=\sum_{n \neq l, m} \frac{\left|\left\langle n\left|H^{\prime}\right| l\right\rangle\right|^{2}}{E_{m}-E_{n}}, \\
\tilde{H}_{m l}=\sum_{n \neq l, m} \frac{\left\langle m\left|H^{\prime}\right| n\right\rangle\left\langle n\left|H^{\prime}\right| l\right\rangle}{E_{m}-E_{n}} .
\end{gathered}
$$

The sum is taken over the remaining nondegenerate states of the bare Hamiltonian. By diagonalizing $\tilde{H}$ and taking its eigenstates as the new basis states for the perturbation calculation, the degeneracy that would otherwise cause the expansions to diverge may be removed.

In the present case each of the degenerate states couples only to $|3,0\rangle$ so that the sums reduce to a single term each and we obtain

$$
\begin{aligned}
\tilde{H}= & \frac{\left|J_{-3}\right|^{2}}{\omega}|-\rangle\left\langle-\left|+\frac{|\lambda|^{2}}{\omega}\right| 3,1\right\rangle\langle 3,1| \\
& +\frac{J_{-3} \lambda}{\omega}(|-\rangle\langle 3,1|+| 3,1\rangle\langle-|),
\end{aligned}
$$

where $J_{-3}=\left\langle 3,0\left|H^{\prime}\right|-\right\rangle=c_{1}^{-} J_{13}+c_{2}^{-} J_{23}$ is the coupling between the state $|-\rangle=c_{1}^{-}|1\rangle+c_{2}^{-}|2\rangle$ and the intermediate state $|3,0\rangle$.

Inspection of Eq. (15) shows that, in fact, $\omega=\epsilon_{-}-\epsilon_{3}$ is not the exact resonance frequency of the interacting system unless $\left|J_{-3}\right|^{2}=|\lambda|^{2}$. The interaction of $|-\rangle(|3,1\rangle)$ with $|3,0\rangle$ shifts its energy, and if the interaction strengths are different the states will be shifted slightly out of resonance. This effect may be accounted for by adding a further perturbation term

$$
H^{\prime \prime}=\delta \omega|3,1\rangle\langle 3,1|
$$

that allows the resonance frequency $\omega$ to be adjusted. This term acts only to second order in the perturbation expansion, so it does not correct the degeneracy to first order. To second order the effective Hamiltonian becomes

$$
\begin{aligned}
\tilde{H}= & \frac{\left|J_{-3}\right|^{2}}{\omega}|-\rangle\left\langle-\left|+\left(\frac{|\lambda|^{2}}{\omega}+\delta \omega\right)\right| 3,1\right\rangle\langle 3,1| \\
& +\frac{J_{-3} \lambda}{\omega}(|-\rangle\langle 3,1|+| 3,1\rangle\langle-|) .
\end{aligned}
$$

The condition for exact resonance is that the diagonal terms of $\tilde{H}$ are equal, which occurs when

$$
\delta \omega=\frac{\left|J_{-3}\right|^{2}-|\lambda|^{2}}{\omega} .
$$

At this point the energy corrections due to interactions within the degenerate subspace are $\tilde{E}_{1}=-\tilde{E}_{2}=J_{-3} \lambda / \omega$ and the original basis states $|-\rangle$ and $|3,1\rangle$ are maximally mixed. To get an idea of how well our degenerate perturbation theory analysis works for the FMO complex, we can compare it against a numerical solution of the full system. The resonance frequencies may be found by using the simulations to identify the avoided crossings of the eigenvalues of $H$ as a function of $\omega$, which gives $\omega \approx 147.1 \mathrm{~cm}^{-1}$ for the lower 
resonance. Perturbation theory predicts a resonance frequency $\omega+\delta \omega=147.1 \mathrm{~cm}^{-1}$, in excellent agreement with the numerical result. Likewise, the energy splitting $2 J_{-3} \lambda / \omega=$ $5.4 \mathrm{~cm}^{-1}$, which agrees well with the numerical value of $5.1 \mathrm{~cm}^{-1}$.

The same analysis may be applied when $\omega$ is such that $|3,1\rangle$ is on resonance with the upper excitonic state $|+\rangle$. A prediction of $\omega+\delta \omega=341.2 \mathrm{~cm}^{-1}$ is obtained. However, in this case the couplings $J_{13}$ and $J_{23}$ add destructively, giving $J_{+3}=-16.8 \mathrm{~cm}^{-1}$, somewhat smaller than $J_{-3}=25.9 \mathrm{~cm}^{-1}$. Together with the larger resonance frequency, this produces an energy splitting of just $0.7 \mathrm{~cm}^{-1}$. Consequentially the effect of the resonance with $|+\rangle$ on the dynamics of the system is much smaller than that of $|-\rangle$. This is not inconsistent with the relaxation pathways proposed by Brixner et al. [37], in which their exciton 7 (roughly equivalent to $|+\rangle$ in our model) decays to exciton $3(|-\rangle$ in our model) rather than directly to the lowest level primarily localized on site 3 . Given the small energy difference and large coupling between sites 1 and 2, this transition seems unlikely to be vibrationally assisted in the coherent sense discussed here. Alternatively, Chin et al. [7] suggest that $|+\rangle$ may couple strongly to one of the exciton states on sites 4-7 of FMO, so it may participate in a more complicated energy-transfer mechanism that is not captured in our simplified model. Therefore we will focus on the resonance with $|-\rangle$ in the dynamical simulations to follow.

Despite its apparent simplicity, the model we constructed captures the interplay between delocalized exciton states and local vibrational modes. It is worth emphasizing that the coupling of the $|-\rangle$ and $|+\rangle$ excitonic states to the vibrationally excited state $|3,1\rangle$ is weak, as is the coupling between $|3,0\rangle$ and $|3,1\rangle$. Nevertheless, when the resonance condition is satisfied the exciton state becomes strongly mixed with the vibrationally excited state, creating delocalized vibronic states. The analytical framework provides both an intuitive picture for how resonance can contribute to energy transport and a solid mathematical method for accurately calculating resonance frequencies and the consequent splitting of the vibronic eigenstates. Within this model we can now study how vibronic resonances together with electronic coupling and environmental noise can contribute to energy-transfer processes.

\section{DYNAMICS OF ENERGY TRANSFER}

\section{A. Coherent dynamics}

Without dephasing or trapping it should be immediately clear that adding a resonant vibrational level provides a dramatic improvement in energy transport across the system. This is illustrated in Fig. 3. In the absence of the vibrational mode, the large energy gap and small coupling between site 3 and the rest of the system produce a high degree of disorder-induced localization. An initial excitation on site 1 primarily oscillates between sites 1 and 2, with the maximum population of site 3 reaching only about $2.5 \%$ [Fig. 3(a)]. Once a vibrational mode is included, our model (17) suggests that an excitation in $|-\rangle$ will be completely transferred to state $|3,1\rangle$ at time $\tau=(\pi / 2)[\hbar \omega /(J \lambda)] \approx 3 \mathrm{ps}$, which is of the right order to be of biological relevance. The corresponding dynamics when the initial excitation is on site 1 is shown in Fig. 3(b). (a)

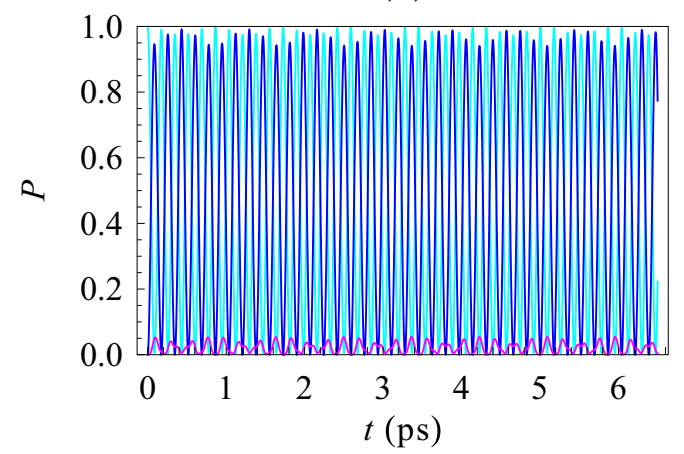

(b)

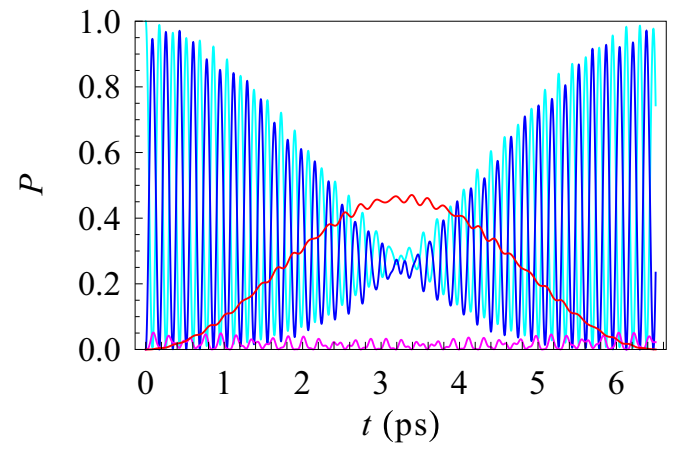

(c)

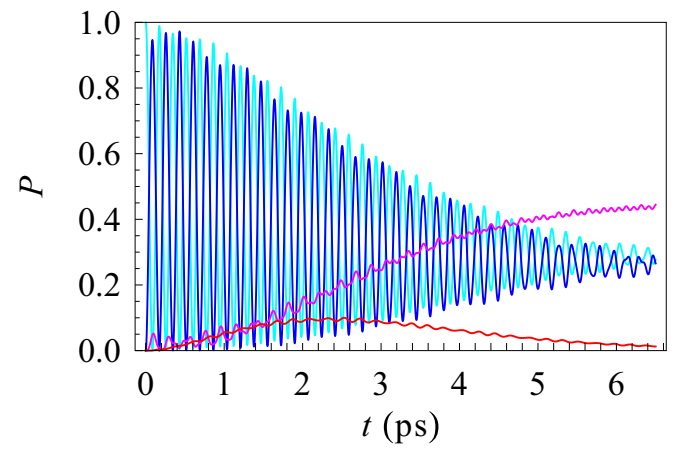

FIG. 3. (Color online) Dynamics of site populations in the absence of dephasing: blue, $|1\rangle$; cyan, $|2\rangle$; magenta, $|3,0\rangle$; red, $|3,1\rangle$. The initial state is $|1\rangle$. (a) Fully coherent dynamics without vibrational coupling $(\lambda=0)$. Disorder-induced localization prevents $|3,0\rangle$ from ever becoming significantly populated. (b) Fully coherent dynamics with a vibrational mode at $147 \mathrm{~cm}^{-1}$ coupled to site 3 , showing resonant oscillations between exciton state $|-\rangle$ and the excited vibrational level $|3,1\rangle$. The absence of vibrational decay means that $|3,0\rangle$ is still not significantly populated. (c) Dynamics including decay of vibrational excitations at rate $\gamma_{\mathrm{vib}}=7.5 \mathrm{~cm}^{-1}$. The initial population in $|-\rangle$ is transferred to $|3,0\rangle$ in roughly $6 \mathrm{ps}$.

The vibrationally excited state $|3,1\rangle$ reaches its maximum population around $3 \mathrm{ps}$, but the vibrational ground state $|3,0\rangle$ is still only slightly populated. Moreover, the dynamics is still fully coherent so the population of $|3,1\rangle$ subsequently transfers back into sites 1 and 2 . Adding decay to the vibrational mode provides directionality of energy transport. On resonance and with $\gamma_{\text {vib }}=7.5 \mathrm{~cm}^{-1}$ (based on the experimental results of Wendling et al. [13]), the population of $|3,0\rangle$ reaches $40 \%$ 


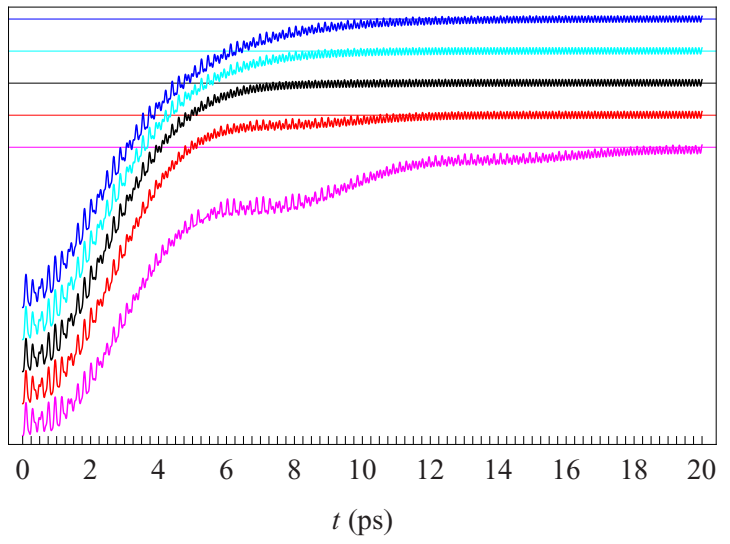

FIG. 4. (Color online) Occupation probability of $|3,0\rangle$ following initial excitation of $|1\rangle$, for various values of the vibrational decay rate; from bottom to top, $\gamma_{\text {vib }}=\{2.5$ (magenta), 5.0 (red), 7.5 (black), 10.0 (cyan), 12.5 (blue) $\} \mathrm{cm}^{-1}$. For clarity, successive curves have been vertically offset by 0.05 ; the corresponding horizontal lines indicate $P=0.45$ for each curve.

after 5 ps following an initial excitation on site 1 [Fig. 3(c)]. In this case the transfer is irreversible. The final population of $|3,0\rangle$ is primarily limited by the amplitude of $|-\rangle$ in the initial state, because $|+\rangle$ couples very weakly to $|3,0\rangle$.

Intriguingly, it turns out that the value of $\gamma_{\mathrm{vib}}=7.5 \mathrm{~cm}^{-1}$ derived from the linewidths found in vibrational spectroscopy of FMO [13] coincides closely with the value that optimizes the speed of population transfer from site 1 to level $|3,0\rangle$. Figure 4 illustrates the effect of changing $\gamma_{\text {vib }}$. To verify that this is not just a coincidence based on the particular choice of site energy and coupling values, we repeated the calculation for a number of data sets from the literature. Typical values for the optimum decay rate are in the range $\gamma_{\text {vib }} \sim 5$ to $7 \mathrm{~cm}^{-1}$, so this result appears to be rather general and robust.

\section{B. Comparison with DAT-ENAQT}

Biological systems, of course, operate under conditions in which dephasing cannot be neglected. Counter to the expectations of many physicists, it has been shown that dephasing can in fact improve energy transport [3-8]. The dephasing mechanism in our model, as in DAT-ENAQT models, corresponds to fluctuations in the excited-state energies of individual chromophores. Unfortunately, this is difficult to relate directly to experimental measurements, because experimentally measured dephasing rates are related to the decay of exciton coherences. Measured values are also affected by the inhomogeneous broadening intrinsic to large samples. As yet, there is no consensus regarding the magnitude of the individual site dephasing rates or their temperature dependence. In our model the situation is further complicated by the inclusion of vibrational modes in the system Hamiltonian, which effectively constitutes a second source of dissipation. We have therefore chosen to approach the problem by taking $\gamma_{\text {deph }}$ as a free parameter and study the dynamics over a range of values.

In order to directly compare the vibrationally assisted system with the results of DAT-ENAQT, a trapping process as

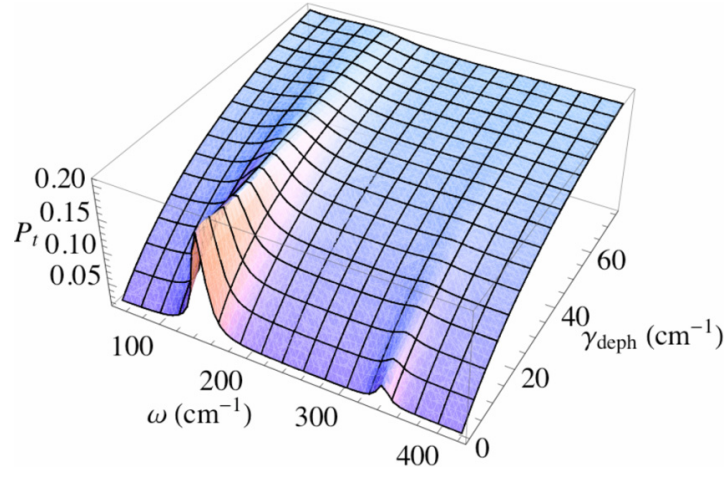

FIG. 5. (Color online) Trap-state population $P_{\mathrm{t}}$ at $5 \mathrm{ps}$ for an initial state on site 1 as a function of vibrational frequency $\omega$ and dephasing rate $\gamma_{\text {deph }}$ (both measured in $\mathrm{cm}^{-1}$ ). The trapping rate has been set to $\gamma_{\text {trap }}=1 \mathrm{~cm}^{-1}$. Peaks at $\omega \approx 147 \mathrm{~cm}^{-1}$ and $341 \mathrm{~cm}^{-1}$ correspond to resonances of $|-\rangle$ and $|+\rangle$ with $|3,1\rangle$.

illustrated in Fig. 1 was added to the general model. Equation (4) was used to calculate the population $P_{\mathrm{t}}$ of the trap site $|t\rangle$ at time $t=5 \mathrm{ps}$, following an initial excitation in state $|1\rangle$. The value of $\gamma_{\text {trap }}=1 \mathrm{~cm}^{-1}=0.03 \mathrm{ps}^{-1}$ was chosen to be similar to that of Refs. [3,4]. Figure 5 shows the results as a function of the vibrational frequency $\omega$ and the dephasing rate $\gamma_{\text {deph }}$. At small dephasing, a significant enhancement of the trap population is found for $\omega \sim 147 \mathrm{~cm}^{-1}$, corresponding to the resonance between $|-\rangle$ and $|3,1\rangle$. A smaller peak due to the resonance with $|+\rangle$ is visible around $\omega=341 \mathrm{~cm}^{-1}$. As the dephasing increases the effect of the resonance diminishes but remains visible up to the highest values considered. This is to be expected: at large dephasing rates, excitonic delocalization is destroyed and the site energy fluctuations become large enough to bridge the large energy gaps on their own, negating the benefit of coherent vibrational coupling.

\section{Dynamics in absence of external trapping}

Irreversible decay to a trapping site is included to ensure efficiency and directionality within pure dephasing models $[3,4,7,8]$. Otherwise, the system evolves smoothly to a steadystate population given by $1 / N$ on each of the $N$ sites of the network [4]. The inclusion of this trapping process is usually justified by the belief that the FMO protein functions as a "molecular wire" that transmits energy from the chlorosome antenna complex to the reaction center [40]. Unfortunately there is very little data about the kinetics of energy transfer from FMO to the reaction center; what few experiments exist have shown surprisingly slow and inefficient transfer, contrary to expectations from structural evidence [41]. Experiments on other antenna-reaction-center complexes have shown that traps spend some fraction of time closed to further energy transfer and also that excitons may have a nontrivial probability of escaping from the trap back into the antenna [1,41]. In FMO neither the trapping rate nor the nature of the trapping process is known. Furthermore, experiments on purified FMO complexes have shown that, even in the absence of the reaction center and its associated trapping process, population is transferred from higher excited states toward the lowest exciton state that is localized primarily on chromophore 3 [37]. Some of the 
(a)

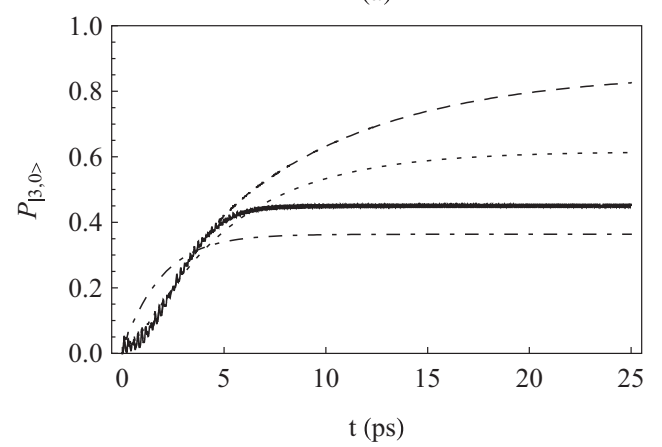

(b)

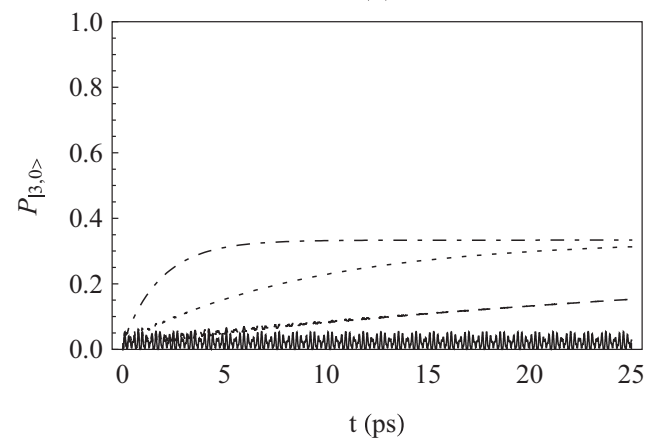

FIG. 6. Population of $|3,0\rangle\left(P_{|3,0\rangle}\right)$ in the absence of trapping for an initial excitation on site 1, both (a) with and (b) without vibrational coupling. Dephasing values (in $\mathrm{cm}^{-1}$ ) are $\gamma_{\text {deph }}=0$ (solid), 2 (dashed), 10 (dotted), and 50 (dot dashed). A 25 ps timescale has been chosen to show the steady-state behavior of the population.

features of the experimental observations are reproduced in simulations with standard thermal techniques using a simple Ohmic spectral density [37], but others are not. In particular, the rate of transfer to the lowest exciton state is calculated to be substantially slower than the observed rate.

By contrast, the vibration-assisted resonance mechanism produces directional energy transfer without an additional trapping process. The lowest energy site serves as the energy "sink," with the decay of the vibrational level providing the asymmetry between forward and backward transfer. Figure 6 shows the population of $|3,0\rangle$ in the absence of trapping (a) with and (b) without vibrational coupling. Without the vibrational coupling, setting $\gamma_{\text {deph }}=0$ demonstrates the poor energy-transport properties of purely coherent dynamics in a disordered network. Finite dephasing values all lead to a steady-state population of $1 / 3$; the larger the dephasing, the faster this state is achieved. In the vibrational case with $\gamma_{\text {deph }}=0$, the steady-state population is determined by the amplitude of $|-\rangle$ in the initial state $|1\rangle$, here nearly one half. Adding a small amount of dephasing allows the initial population in $|+\rangle$ to be transferred with high probability. However, increasing the dephasing beyond a certain optimal value (here around $2 \mathrm{~cm}^{-1}$ ) reduces the population transfer. At sufficiently large dephasing values the diffusion limit is recovered, in which the population of $|3,0\rangle$ rises quickly to its steady-state value. The vibration-assisted increase in transfer efficiency appears to be quite sensitive to the precise dephasing rate and approaches its maximum value over a relatively long timescale. On the other hand, the improvement is caused by the inclusion of a dissipative nuclear vibrational mode, a process that has a sound physical basis. Although the calculations shown here are restricted to a zero-temperature mode, we have verified that there is no qualitative change in the dynamics over the range of temperatures over which our simplified model remains valid.

Recent numerical calculations [20,21] using the nonperturbative hierarchical equations of motion method have been carried out using peaked spectral densities that closely approximate the experimental results of Wendling et al. [13]. Their results suggest that the pure dephasing rate may be much smaller than previously believed [3,4,6]. Compared to typical smooth spectral densities with a large pure dephasing rate (see, for instance, Ref. [27]), the spectral densities modeled on the experiments of Wendling et al. [13], with large values at frequencies close to those of the excitonic transitions, lead to faster energy transfer through the FMO complex. These models emphasize the importance of relaxation processes at the excitonic transitions over the role of pure dephasing. Furthermore, the multipeaked spectral densities produce theoretical predictions that are in better agreement with the experimental results from two-dimensional (2D) spectroscopy. Our findings similarly suggest that fast and efficient transfer can be achieved through coupling to a resonant, damped vibrational mode together with a small amount of pure dephasing. The connection can be made more precise by considering pseudomode $[42,43]$ or Fano theory [44], in which a thermal bath with a Lorentzian spectral density is shown to be formally equivalent to a damped oscillator mode incorporated in the system Hamiltonian. Accordingly, it should not be necessary to have a sharp peak near the resonance; a continuous spectral density with a large amplitude near the transition frequency should display the same physics, as shown by the comparison of the $J_{3 \text { peaks }}$ and $J_{11 \text { peaks }}$ spectral densities in Ref. [20]. Our model thus provides an intuition into the microscopic physical mechanism by which the modes near the excitonic transitions contribute to energy transfer.

\section{DISCUSSION AND CONCLUSIONS}

Drawing on physical intuition from incoherent FRET theory, we proposed a general model for energy-transport dynamics in the coherent regime where internal exciton couplings exceed the interaction with the thermal environment. Incorporating resonant vibrational modes into the system Hamiltonian provides a physically motivated mechanism for simultaneously providing directionality to the energy transport and improving its efficiency over pure-dephasing models. This mechanism does not require the assumption of external energy trapping, individually optimized site-dephasing rates, or correlated bath fluctuations.

Analysis of a simple case based on the FMO complex clearly shows that it is only the resonant vibrational levels that significantly affect the dynamics. Interactions with offresonant modes renormalize the exciton energies, altering the resonance frequencies of the network but not the character of the eigenstates. This insight suggests a method for applying the 
general model to specific systems of interest. First, resonances in the excitonic system are identified by using perturbation theory as a guide. Only resonant vibrational levels need be included in the system Hamiltonian along with the electronic energies and couplings. Dynamics can then be calculated using a Lindblad master equation that incorporates both electronic dephasing and decay of vibrational excitations. While it is not possible to directly compare the simplified model of FMO treated here with experimental results, a few remarks are in order.

Comparison of the resonance frequencies obtained in our three-site model with the vibrational frequencies obtained in the experiments of Wendling et al. [13] shows that our calculated frequencies fall into gaps in the vibrational spectrum. To a certain extent this is because we consider only three sites and a single mode; coupling to the remaining sites and modes provides further shifts to the exciton levels $| \pm\rangle$, which will alter the values of $\omega$. More fundamentally, the resonance frequency depends strongly on the particular values chosen for the site energies and exciton couplings. As yet there is no direct experimental method for measuring the site energies, and values based on theoretical fits to experimental spectra vary considerably [9]. In order to estimate the sensitivity of the frequency to changes in the Hamiltonian, we calculated resonance frequencies from a number of published data sets for the site energies and exciton couplings in the FMO complex of $P$. aestuarii. Values for the lower resonance ranged from 94 to $167 \mathrm{~cm}^{-1}$. From this exercise we conclude that the site energies, in particular, of FMO are not yet sufficiently well known to enable accurate calculation of resonance frequencies.

Recent experiments have been designed to address the hypothesis that vibrational and/or vibronic coherence might be responsible for the observed quantum beating in FMO. Hayes et al. studied FMO complexes with modified vibronic structure and found little effect on the frequency or dephasing of the beating in the exciton 1-2 crosspeak [45]. The beat frequency is equal to the energy difference between the two exciton levels. In our model, resonance with the vibrational mode has little effect on the energy difference since the splitting due to the resonance is small, about $5 \mathrm{~cm}^{-1}$. Therefore, we would not expect to observe much change in the coherence beat frequency when the vibrational mode is shifted off resonance. The primary effect of the resonance is an enhancement of the energy-transfer efficiency, which was not addressed in that particular experiment. Another experiment designed to compare the 2D spectra of $\mathrm{BChl} a$ in solution with the spectra of FMO was unable to resolve more than two vibrational modes, which had frequencies well above those relevant for our model [46]. Neither of these experiments would seem to rule out a vibration-assisted resonance mechanism.

On the other hand, an experiment examining the inhomogeneous broadening of the exciton 1-3 coherence in FMO found the energy gap between the exciton levels to be quite consistent across the sample [47]. This is not necessarily a general feature of photosynthetic pigment-protein complexes, because the authors also studied the LH2 antenna complex from purple bacteria and found a large degree of inhomogeneous broadening. Their result supports the idea that having a well-defined exciton splitting is important to the functioning of the FMO complex. Such an interpretation would be more consistent with our model, which requires a close match between the exciton splitting and a specific vibrational frequency, than with the dephasing-assisted mechanism that places no restrictions on the exciton splittings.

Finally, we speculate that the vibration-assisted resonance mechanism may be able to shed some light on the current controversies over experiments demonstrating long-lived coherence oscillations in the FMO complex. The model suggests that the intramolecular components of the vibrational spectral density can enhance coherent transport rather than destroying it. This is in contrast to the conclusions of Christensson et al. [48]. The vibronic theory of Refs. [26,48] neglects noise effects, including damping, on the vibrational mode and focuses on vibrational coherences that are localized on a single pigment. Our results show that the resonance-induced delocalization of the mixed vibronic-excitonic states and the decay of vibrational excitations are key to the enhancement of transport. Indeed, by strongly mixing a vibrationally excited level on one site with the vibrational ground state of another site, vibration-assisted resonance creates an even greater degree of coherent delocalization across the network than excitonic coupling alone. More significantly, the microscopic mechanism presented here may be able to conceptually reconcile the large reorganization energies and lack of correlated energy fluctuations found in atomistic numerical studies [17,18,29] with experimental observations showing the persistence of coherent oscillations up to physiological temperatures [49]. Our model shows some suggestive similarities with nonperturbative numerical calculations that demonstrate the role of a strongly peaked spectral density in reconciling strong environmental coupling with persistent coherence [20,21]. Further theoretical work on the full FMO complex with multiple vibrational modes, as well as improved experimental characterization of the site energies, kinetics, and decoherence properties of pigment-protein complexes, will be needed in order to determine whether coherent vibration-assisted resonance plays a significant role in biological function.

\section{ACKNOWLEDGMENTS}

Helpful discussions with Aggie Brańczyk, Simon Benjamin, Erik Gauger, Kieran Higgins, Felix Pollock, Amir Fruchtman, Vaia Patta, and Elliott Levi are gratefully acknowledged. B.W.L. thanks the Royal Society for support from a University Research Fellowship. This work was funded by the Leverhulme Trust.
[1] R. E. Blankenship, Molecular Mechanisms of Photosynthesis (Blackwell Publishing, Oxford, 2002).

[2] D. L. Andrews and J. Rodríguez, J. Chem. Phys. 127, 084509 (2007).
[3] M. B. Plenio and S. F. Huelga, New J. Phys. 10, 113019 (2008).

[4] P. Rebentrost, M. Mohseni, I. Kassal, S. Lloyd, and A. AspuruGuzik, New J. Phys. 11, 033003 (2009).

[5] J. A. Leegwater, J. Phys. Chem. 100, 14403 (1996). 
[6] F. Caruso, A. W. Chin, A. Datta, S. F. Huelga, and M. B. Plenio, J. Chem. Phys. 131, 105106 (2009).

[7] A. W. Chin, A. Datta, F. Caruso, S. F. Huelga, and M. B. Plenio, New J. Phys. 12, 065002 (2010).

[8] A. W. Chin, S. F. Huelga, and M. B. Plenio, Philos. Trans. R. Soc., A 370, 3638 (2012).

[9] M. T. W. Milder, B. Brüggemann, R. van Grondelle, and J. L. Herek, Photosynth. Res. 104, 257 (2010).

[10] E. Rivera, D. Montemayor, M. Masia, and D. F. Coker, J. Phys. Chem. B 117, 5510 (2013).

[11] I. Rips, Phys. Rev. E 47, 67 (1993).

[12] S. Mukamel, Principles of Nonlinear Optical Spectroscopy (Oxford University Press, Oxford, UK, 1995).

[13] M. Wendling, T. Pullerits, M. A. Przyjalgowski, S. I. E. Vulto, T. J. Aartsma, R. van Grondelle, and H. van Amerongen, J. Phys. Chem. B 104, 5825 (2000).

[14] M. Rätsep and A. Freiberg, J. Lumin. 127, 251 (2007).

[15] A. B. Doust, C. N. J. Marai, S. J. Harrop, K. E. Wilk, P. M. G. Curmi, and G. D. Scholes, J. Mol. Biol. 344, 135 (2004).

[16] V. I. Novoderezhkin, A. B. Doust, C. Curutchet, G. D. Scholes, and R. van Grondelle, Biophys. J. 99, 344 (2010).

[17] C. Olbrich, J. Strümpfer, K. Schulten, and U. Kleinekathöfer, J. Phys. Chem. Lett. 2011, 1771 (2011).

[18] S. Shim, P. Rebentrost, S. Valleau, and A. Aspuru-Guzik, Biophys. J. 102, 649 (2012).

[19] G. Ritschel, J. Roden, W. T. Strunz, and A. Eisfeld, New J. Phys. 13, 113034 (2011).

[20] C. Kreisbeck and T. Kramer, J. Phys. Chem. Lett. 3, 2828 (2012).

[21] C. Kreisbeck, T. Kramer, and A. Aspuru-Guzik, J. Phys. Chem. B 117, 9380 (2013).

[22] M. del Rey, A. W. Chin, S. F. Huelga, and M. B. Plenio, J. Phys. Chem. Lett. 4, 903 (2013).

[23] A. W. Chin, J. Prior, R. Rosenbach, F. Caycedo-Soler, S. F. Huelga, and M. B. Plenio, Nat. Phys. 9, 113 (2013).

[24] J. Lim, M. Tame, K. H. Yee, J.-S. Lee, and J. Lee, New J. Phys. 16, 053018 (2014).

[25] A. Kolli, E. J. O'Reilly, G. D. Scholes, and A. Olaya-Castro, J. Chem. Phys. 137, 174109 (2012).

[26] A. Chenu, N. Christensson, H. F. Kauffmann, and T. Mančal, Sci. Rep. 3, 2029 (2013).

[27] A. Ishizaki and G. R. Fleming, Proc. Natl. Acad. Sci. USA 106, 17255 (2009).
[28] P. Nalbach, A. Ishizaki, G. R. Fleming, and M. Thorwart, New J. Phys. 13, 063040 (2011).

[29] C. Olbrich, T. L. C. Jansen, J. Liebers, M. Aghtar, J. Strümpfer, K. Schulten, J. Knoester, and U. Kleinekathöfer, J. Phys. Chem. B 115, 8609 (2011).

[30] T. Renger, V. May, and O. Kühn, Phys. Rep. 343, 137 (2001).

[31] T. Soules and C. Duke, Phys. Rev. B 3, 262 (1971).

[32] F. L. Semião, K. Furuya, and G. J. Milburn, New J. Phys. 12, 083033 (2010).

[33] N. J. Turro, J. C. Scaiano, and V. Ramamurthy, Modern Molecular Photochemistry of Organic Molecules (University Science Books, Sausalito, CA, 2010).

[34] M. Rätsep, Z.-L. Cai, J. R. Reimers, and A. Freiberg, J. Chem. Phys. 134, 024506 (2011).

[35] H.-P. Breuer and F. Petruccione, The Theory of Open Quantum Systems (Oxford University Press, Oxford, UK, 2002).

[36] J. Adolphs and T. Renger, Biophys. J. 91, 2778 (2006).

[37] T. Brixner, J. Stenger, H. M. Vaswani, M. Cho, R. E. Blankenship, and G. R. Fleming, Nature (London) 434, 625 (2005).

[38] J. M. Jean, R. A. Friesner, and G. R. Fleming, J. Chem. Phys. 96, 5827 (1992).

[39] L. I. Schiff, Quantum Mechanics, 3rd ed. (McGraw-Hill, New York, 1968).

[40] J. M. Olson, Photosynth. Res. 80, 181 (2004).

[41] J. Amesz and S. Neerken, Photosynth. Res. 73, 73 (2002).

[42] B. M. Garraway, Phys. Rev. A 55, 2290 (1997).

[43] B. M. Garraway, Phys. Rev. A 55, 4636 (1997).

[44] J. Roden, W. T. Strunz, K. B. Whaley, and A. Eisfeld, J. Chem. Phys. 137, 204110 (2012).

[45] D. Hayes, J. Wen, G. Panitchayangkoon, R. E. Blankenship, and G. S. Engel, Faraday Discuss. 150, 459 (2011).

[46] K. A. Fransted, J. R. Caram, D. Hayes, and G. S. Engel, J. Chem. Phys. 137, 125101 (2012).

[47] A. F. Fidler, E. Harel, P. D. Long, and G. S. Engel, J. Phys. Chem. A 116, 282 (2012).

[48] N. Christensson, H. F. Kauffmann, T. Pullerits, and T. Mančal, J. Phys. Chem. B 116, 7449 (2012).

[49] G. Panitchayangkoon, D. Hayes, K. A. Fransted, J. R. Caram, E. Harel, J. Wen, R. E. Blankenship, and G. S. Engel, Proc. Natl. Acad. Sci. USA 107, 12766 (2010). 